\title{
Targeted disruption of EBNA1 in EBV-infected cells attenuated cell growth
}

\author{
Ka-Won Noh ${ }^{1, \#}$, Jihyun Park ${ }^{1, \#} \mathcal{E}$ Myung-Soo Kang ${ }^{1,2,3, *}$ \\ ${ }^{1}$ Department of Health Sciences and Technology, Samsung Advanced Institute for Health Sciences and Technology (SAIHST), \\ Sungkyunkwan University, ${ }^{2}$ Samsung Biomedical Research Institute (SBRI), Samsung Medical Center, Seoul 06351, ${ }^{3}$ BioMembrane \\ Plasticity Research Center (MPRC), Seoul National University College of Medicine, Seoul 03080, Korea
}

\begin{abstract}
Epstein Barr virus (EBV)-encoded nuclear antigen-1 (EBNA1) plays a pivotal in an EBV episome replication and persistence. Despite considerable attempts, there are no EBV drugs or vaccines. We attempted to eradicate EBV episomes by targeting EBNA1 using the transcription activator-like effector nucleases (TALEN) (E1TN). E1TN-mediated transient knockout (KO) of EBNA1 reduced EBNA1 expression, and caused significant loss of EBV genomes and progressive death of EBV-infected cells. Furthermore, when a mixture of EBV-infected Burkitt's lymphoma (BL) cells and EBV-negative BL cells was targeted by E1TN, EBV-negative cells were counter-selected while most EBV-infected cells died, further substantiating that EBNA1 KO caused selective death of EBV-infected cells. TALEN-mediated transient targeting of EBNA1 attenuated the growth of EBV-infected cells, implicating a possible therapeutic application of E1TN for EBV-associated disorders. [BMB Reports 2016; 49(4): 226-231]
\end{abstract}

\section{INTRODUCTION}

Epstein-Barr virus (EBV) establishes lifelong latency in its hosts by undergoing latent replication and persistence of viral episomal genomes. EBV infection is considered a high risk factor and is responsible for $>1 \%$ of all human cancers (1). Although the synthesis of viral genome is initiated by the host replication machinery, efficient replication and long term persistence require EBV nuclear antigen 1 (EBNA1)-mediated DNA binding activity (2). In latent infections, including malignancies, EBV usually persists as a multi-copy episome (3, 4). EBV genome integration is unusual $(5,6)$. EBNA1 is essential

*Corresponding author. Tel: +82-2-740-8919; Fax: +82-2-740-8918; E-mail: emeskang@gmail.com

${ }^{\text {"}}$ These authors contributed equally to this work.

http://dx.doi.org/10.5483/BMBRep.2016.49.4.260

Received 18 December 2015, Revised 11 January 2016, Accepted 15 February 2016

Keywords: Cell death, EBNA1, Epstein-Barr Virus, Knockout, TALEN for EBV episome persistence in dividing cells and enhances episome transcription $(7,8)$, validating the status of EBNA1 as a reasonable target protein for the treatment of EBV-associated diseases (9-12). EBV genome has 24 cognate sites in OriP element where EBNA1 binds, enabling EBV genome replication and transcription. Transcription activator-like effector (TALE)based nuclease (TALEN) can introduce frame-shift mutation in specific genomic sites (13). Despite considerable attempts towards developing EBV drugs $(9,14,15)$, there is no FDA-approved EBV drugs or vaccines (16). Thus, we have attempted to target EBNA1 in living cells by using TALEN system (E1TN) to assess the potential therapeutic effect of E1TN on the growth of EBV-infected gastric cancer (GC), Burkitt's lymphoma (BL), and lymphoblastoid cell line (LCL) $(13,17-19)$.

\section{RESULTS}

Transient EBNA1 KO caused selective cell death in EBV-infected cells

Given that EBNA1 is essential for EBV extra-chromosomal genome replication and persistence and that EBV genome endurance is usually required for EBV-infected cell growth, we hypothesized that targeted transient disruption of EBNA1 by E1TN pair will cause a selective death or attenuation of EBV-infected cells but not EBV-negatives in vitro. To this end, we transiently targeted EBNA1 by transfecting EBV-infected cells (therefore EBNA1-postive) with pair of EBNA1 targeting E1TN vectors and surrogate EBNA1 marker reporter of red fluorescence protein / green fluorescence protein (RFP ${ }^{+} / \mathrm{GFP}^{-@ E B N A 1}$ ) at 1:1:2 ratio, sorted double negative and double positive for RFP/GFP, plated in a 96-well plate at various densities (1, 10, $20,50,100,200,500$ cells per well depending on cell types), and scored the number of wells where cells outgrew at 2 weeks post plating (Table S1, Fig. 1A, B). Those $\mathrm{RFP}^{+} / \mathrm{GFP}^{+}$cells were assumed to have gone through E1TN pair-induced frame shift mutation(s) on targeting site in surrogate $\mathrm{RFP}^{+} / \mathrm{GFP}^{-@ E B N A 1}$ reporter at least (likely also on endogenous EBNA1 site) while $\mathrm{RFP}^{-} / \mathrm{GFP}^{-}$cells were ones where reporter was un-transfected (Fig. 1B). The RFP ${ }^{+} / \mathrm{GFP}^{-@ E B N A 1}$ reporter plasmid constitutively expresses RFP when the GFP sequence is bound to the RFP sequence out of frame. GFP expression is recovered by

ISSN: 1976-670X (electronic edition)

Copyright (c) 2016 by the The Korean Society for Biochemistry and Molecular Biology

(c) This is an open-access article distributed under the terms of the Creative Commons Attribution Non-Commercial License (http://creativecommons.org/licenses/by-nc/4.0) which permits unrestricted non-commercial use, distribution, and reproduction in any medium, provided the original work is properly cited. 


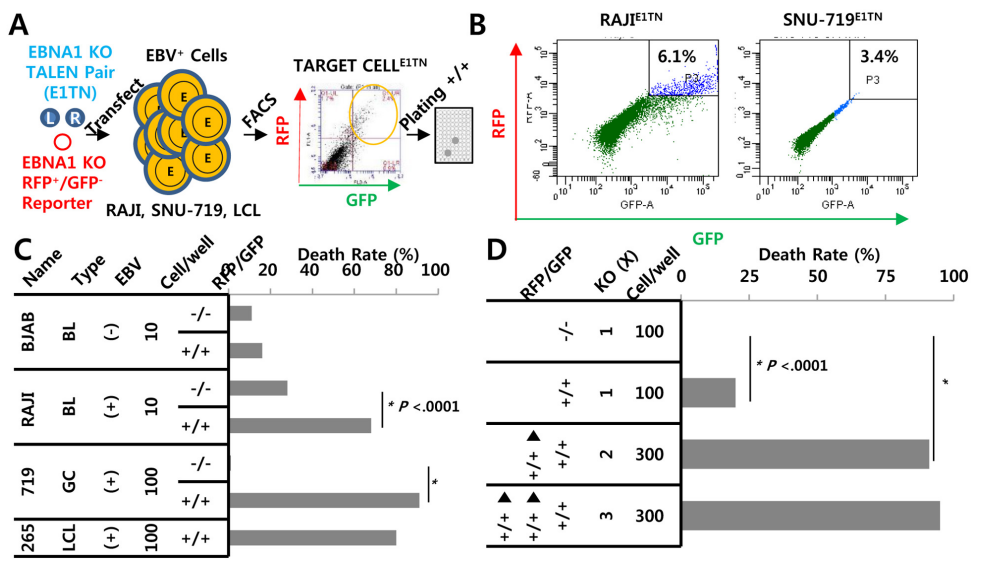

Fig. 1. Transient knock-out $(\mathrm{KO})$ of EBNA1 in EBV-infected cancer cells caused substantial cell death. (A) Experimental scheme of TALEN-mediated KO of EBNA1. RFP/GFP double positive cells were sorted by FACS at 48 hr-post transfection and plated in 96 well plates at various cell densities of $1,10,100,1000$ cells/well. Three weeks after plating, the number of outgrown wells was scored. (B) Approximately $6.1 \%$ and $3.4 \%$ cells showed double positivity in EBNA1-targeted RAJI ${ }^{\mathrm{E} 1 \mathrm{TN}}$ and SNU-719 ${ }^{\mathrm{E} 1 \mathrm{TN}}$ cells. The percentage of strong RFP + GFP + cells was usually less than that observed by the fluorescent microscope. (C) Single transfection of the E1TN pair induced a death of EBV-positive RAJI Burkitt's lymphoma (BL), SNU-719 gastric carcinoma (GC) and SNU-265 lymphoblastoid cell line (LCL) cells but not in EBV-negative BJAB BL cells. Double-positive or double-negative cells were plated at 10 cells of BAJB bad RAJl per well or 100 cells of SNU-719 and SNU-265 per wells for SNU-719 and SNU-265. E1TN pair did not show significant toxicity as observed from similar cell plating efficiency. E1TN pair mediated transient KO of EBNA1 in EBV-infected RAJI BL and SNU-719 GC cell line induced progressive cell death. $\mathrm{P}$ value by Student's t-test $\left({ }^{*} \mathrm{P}<.0001\right)$. (D) Second transfection of E1TN pair resulted in a significant decrease in the plating efficiency of RAJI cells, likely inducing progressive cell death. Upon first round of transfection (denoted as 1 in the number of E1TN pair transfection), all double negative cells (non-transfected) grew rapidly while double positive cells died at a rate of $20 \%$. Upon second and third rounds of transfections into selected and outgrown colonies of RAJl cells from the first round, cells in most wells (85\% and $95 \%$, respectively) died when plated at a density of 100 cells/well.

frame shift mutation via error-prone non homologous end joining (NHEJ) only when the potential target site is disrupted.

In support of the hypotheses, plating $\mathrm{RFP}^{+} / \mathrm{GFP}^{+} \mathrm{RAJl}$ at 1 , 10 , and 100 cells/well upon EBNA1 transient disruption resulted in 100,68 , and $20 \%$ of cell death, respectively. In contrast, plating the $\mathrm{RFP}^{-} / \mathrm{GFP}^{-} \mathrm{RAJl} \mathrm{BL}$ control fraction at 1,10 and 100 cells/well gave rise to $90,28,0 \%$ of cell death in total wells, respectively, as expected; this was indicative of the significantly higher rate of cell death in EBNA1-targeted cells (RFP+/GFP+) than in EBNA1-non-targeted cells (RFP-/GFP-) $(\mathrm{P}<$.0001) (Fig. 1C, D, Table S1). The effect was even more striking in the adherent SNU-719 cells, a naturally EBV-infected GC cell line, as plating RFP+/GFP+ cells at less than or equal to 100 cells per well resulted in no or almost no outgrowth. For 500 cells/well, only $20 \%$ grew from all the wells (Table S1, Fig. 1C). We have observed essentially similar results from the SNU-265 cell line, a naturally EBV infected $\mathrm{LCL}$; Plating of EBNA1-targeted, hence $\mathrm{RFP}^{+} / \mathrm{GFP}^{+}$, SNU-265 $\mathrm{LCL}$ cells at a density of $<50$ cells/well gave rise to no outgrowth. The possibility that E1TN pair is toxic to cells was ruled out in the experiment using EBV-negative $B J A B$ cells. Transfection of the E1TN pair into BJAB BL (hence EBNA1negative) did not affect cell growth in the aforementioned experiment (Table S1, Fig. 1C). These results indicate that E1TN-mediated transient $\mathrm{KO}$ of EBNA1 in EBV-infected cells is sufficient for the attenuation or death of EBV-positive cells with no discernible toxicity.

\section{Repeated, transient transfection of E1TN pair induced progressive EBV + RAJl cell death and transient EBNA1 KO caused decrease in EBNA1 level and EBV genome copy number}

To substantiate the negative role of E1TN pair in EBV-infected cell growth, RFP/GFP double positive cells from the first transfected RAJI were grown until all cells were verified to no longer carry E1TN by fluorescence-activated cell sorting (FACS) analyses (note that the reporter plasmid and E1TN pair plasmids do not carry antibiotics-resistance markers). The subpopulations were brought up transfected, plated at a density of 300 cells/well in 96-well plate, and scored for cell survival as described above during 2 weeks post plating. As a result, further $\mathrm{KO}$ by the second transfection of E1TN resulted in progressive cell death in 87 of 96 wells (Table S2, Fig. 1D). Western blotting analyses to EBNA1 monoclonal antibody verified the decrease in EBNA1 from first (Fig. 2A, B, single transfection) and successive second rounds of E1TN pair transfection (Fig. 2C, 3 rounds of transfection) in type III latent RAJI and type I latent SNU-719, both of which are naturally EBV-infected cancer cells. In RAJI cells showing type III EBV latency in which EBNA2 and LMP1 are also expressed from episomal 
A

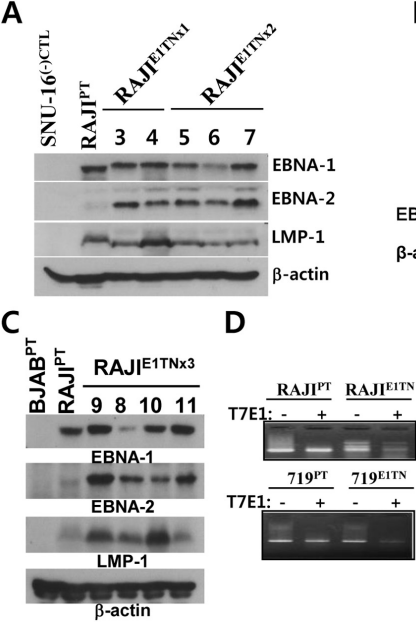

B

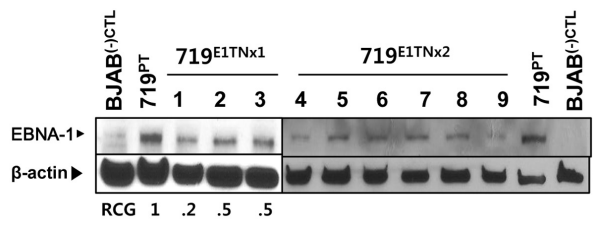

E

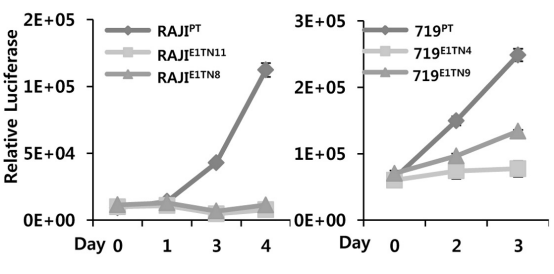

Fig. 2. Repeated, transient transfection of E1TN pair caused the decrease in EBNA1 level and growth attenuation of EBV-infected cells. (A, B) Western blotting (WB) to EBNA1, EBNA2, LMP1 and $\beta$-actin in the clones (shown in Fig. 1C or D) of RAJl cells with type III latency (A) and to EBNA1 in SNU-719 cells with type I latency (B). Note there was more robust knock-down (KD) in EBNA1 by the second round $(\mathrm{E} 1 \mathrm{TN} \times 2)$ than by the first round targeting $(\mathrm{E} 1 \mathrm{TNx} 1)$ in both RAJI and SNU-719. The relative cell growth (RCG) of EBV ${ }^{\text {low }}$ (therefore EBNA $1^{\text {low }}$ ) clones was typically between $10 \%$ and at most $50 \%$ in vitro (denoted as .1 to .5) (see RCG). (C) Third round and repeated transfection of E1TN (RAJI ${ }^{\text {ETN } 3}$ ) caused more significant, but incomplete, loss of EBNA1. Note the low expression of EBNA2 and LMP1 in RAJI in panel A and C likely results from unusual experimental deviation. (D) The target region of EBNA1 was PCR-amplified, denatured, annealed, and digested with T7 endonuclease 1 (T7E1).The appearance of shorter bands or disappearance of expected DNA bands indicates E1TN pair-mediated occurrence of deletion or frame-shift mutation in the target site of EBNA1. (E) In vitro cell growth attenuation

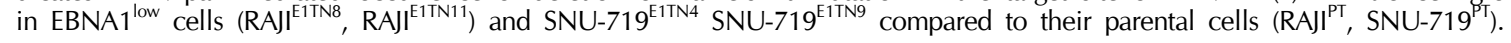

EBV genomes, EBNA1 targeting resulted in concomitant decrease in the EBNA2 but less robustly in LMP1 (Fig. 2A, C). Surviving colonies (RAJl ${ }^{\mathrm{E} T \mathrm{TN}-5}$, SNU-719 ${ }^{\mathrm{E} 1 \mathrm{TN}-4}$ ) were verified to have a "cut and rejoin in the target site", which likely resulted from a frame shift event as shown by T7E1 assay (Fig. 2D); therefore, lower level of EBNA1 was noted. Although insertion or deletions (indels) cannot be observed with the naked eye, the faded bands after T7E1 treatment imply that TALEN worked and did partially delete EBNA1. RAJI ${ }^{\text {ETN-5 }}$ cells were further propagated, re-transfected, plated at 300 cells/well in 96-well plate, and scored for cell survival in 2 weeks after plating in the same way. This repeated KO of EBNA1 then again induced progressive cell death in $96 \%$ of wells. However, ultimately no derivation of EBV-eliminated RAJl cells suggests that the persistence of EBV episome by the virtue of functional EBNA1 is essential for prolonged growth of EBV-infected cells in vitro (Table S2). EBNA1 expression was significantly lower in several clones that survived from multiple rounds of E1TN pair transfection (RAJl ${ }^{\mathrm{E} 1 \mathrm{TN}}$ in Fig. 2A and SNU-719 ${ }^{\mathrm{E} 1 \mathrm{TN}}$ in Fig. 2B). In accordance with the hypothesis, E1TN-targeted EBV low (therefore $\mathrm{EBNA} 1^{\text {low }}$ ) clones grew at a much slower rate between $10 \%$ and $50 \%$ in vitro (Fig. 2A, B, see the relative cell growth (RCG) under panel pictures).

EBNA1 KO counter-selected EBV-negative cells from the pre-mixtures of EBV-negative and EBV-infected cells

The failure to derive EBV-eliminated yet live cells validates the requirement of EBV genome for cell growth and survival. Therefore, we performed spike experiments in an attempt to check whether transient EBNA1 KO can counter select EBVnegative cells from a mixture of EBV-negative and infected cells. To support this idea, we premixed EBV-negative BJAB and EBV-infected RAJl cells at $1: 10^{3}, 10^{2}$ and 10 ratios, which were then followed by the transfection of $\mathrm{RFP}^{+} / \mathrm{GFP}^{-@ E B N A 1}$ reporter and E1TN pair in the same method as mentioned in Fig. 3A. These resulting surviving clones were propagated and 12 randomly selected clones were subjected to FGA short tandem repeat analyses using $B J A B$ and RAJl as the references. As a result, the higher number of spiked $B J A B$ cells, the more $B J A B$ cells were counter-selected (Table S3, Fig. 3B); Two, six and nine clones of 12 randomly selected clones from 1:1000, $1: 100$ and $1: 10$ spiked ratio, respectively, were identified as $B J A B$ cells. A spike ration of $1: 1000$ of $B J A B$ : RAJl induced the survival ratio of 84 from 88 wells and short tandem repeats (STR) analyses with 12 randomly selected clones revealed 2 $B J A B$ cell line (Table S3) (STR data not shown). In the next spiking experiment where 10-fold BJAB cells were premixed with RAJl cells (BJAB: RAJl at 1:100 ratio), 23 of 30 wells were selected $(77 \%)$ and STR analyses for randomly selected 12 colonies verified that a higher number of $B J A B(6 / 12,50 \%)$, and a concomitantly less number of RAJI $(5 / 12,42 \%)$ cells, were selected as expected (Fig. 3C). Identity was further verified by comprehensive STR analyses using 16 markers (Fig. 3D). Furthermore, spiking of $B J A B$ with RAJl cells at a ratio of $1: 10$ 
A



B

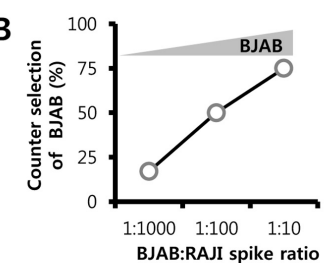

C

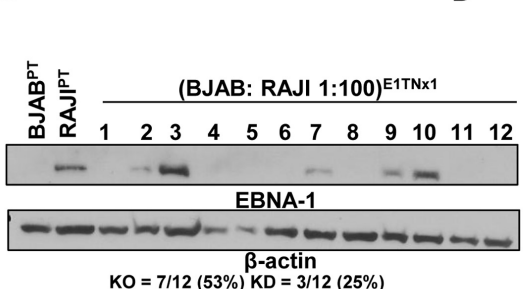

点 点 (BJAB: RAJI, 1:100) E1TNx1
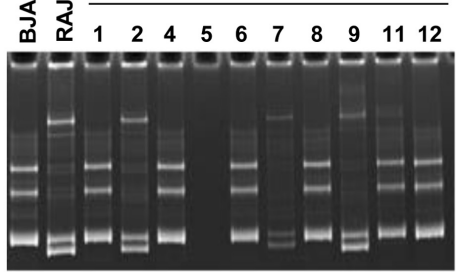

Fig. 3. Transient $\mathrm{KO}$ of EBNA1 in spiked cells counter-selected most EBV-negative BJAB cells. (A) Experimental scheme of TALEN-mediated $\mathrm{KO}$ of EBNA1. EBVnegative $B J A B$ and EBV-infected RAJI cells were mixed at a ratio of $1: 3-10^{3}$ ratios, transiently transfected, sorted, and selected as described above (B) Higher counter-selection of EBV-negative BJAB cells from the cell mixture with higher $\mathrm{BJAB}$ to RAJI. (C, D) Western blotting analysis (C) to EBNA1 and beta-actin antibody and short tandem repeat (STR) analyses (D) for the randomly selected 12 clones from the 1:100 BJAB:RAJI spiked experiment. WB revealed that two clones (clone 3 and 10) were RAJI cells and therefore these two were not subjected to STR.

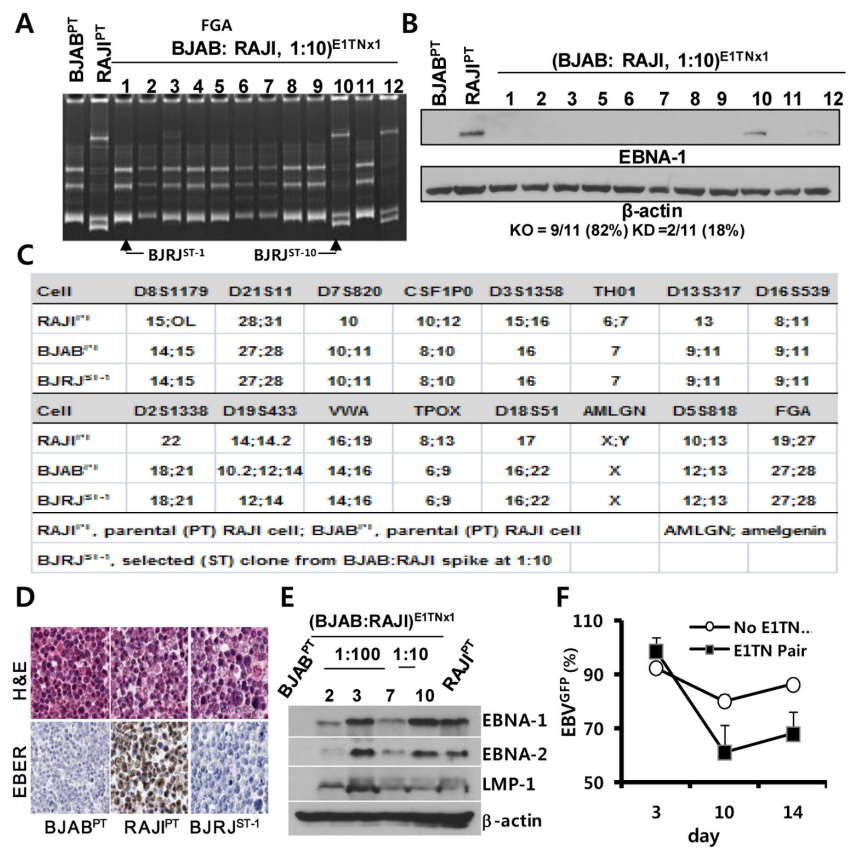

Fig. 4. Transient transfection of E1TN pair induced progressive loss of EBV episome from EBV-infected BL cells. (A, B) Short tandem repeat (STR) analyses (A) and Western blotting analysis (B) for randomly selected 12 clones from 1:10 BJAB:RAJI spiked experiment. While $50 \%$ the surviving clones were identified as BJAB from the 1:100 (BJAB: RAJI) spiked cells, $75 \%$ were identified as BJAB from the 1:10 spiked experiment, indicating that the higher number of BJAB cells were spiked with less RAJI admixture, the higher the counter selection of BJAB cells. (C) STR analyses of two representative clones selected from the BJAB:RAJI mixture (BJRJ ${ }^{\mathrm{ST}-1}$ and BJRJ ${ }^{\mathrm{ST}-10}$ presumed as BJAB and RAII, respectively, from the mini STR. The comprehensive STR results for 16 STR markers were in accordance to the results of FGA STR in (A) and EBNA1 WB in (B). (D) EBV negativity in BJRJ ${ }^{\mathrm{ST}-1}$ as shown by EBER in situ staining. Note that the negative control BJAB cells and positive control RAJl cells stained as expected, (E) EBNA1 KD was collinear to EBNA2 and LMP1 KD in clones 2, 3, 7 shown in Fig. $3 \mathrm{C}$ and Fig. 4B. (F) Single transient transfection of the E1TN pair induced progressive loss of EBV episome from EBV-infected BL cells. EBV $+\mathrm{BJAB}$ cells carrying latent $\mathrm{EBV}^{\mathrm{GFP}}$ genomes were transfected once with the RFP+/GFP-@EBNA1 reporter and E1TN pair. The progressive loss of EBV was presented with the loss of GFP. Note that the transient single transfection induced EBV loss by $39 \%$ to $32 \%$.

resulted in partial growth in 52 wells out of 96 plated wells. STR analysis of randomly selected 12 wells showed that majority of the survived colonies $(9 / 12,75 \%)$ were BJAB cells and only 2 of them $(2 / 12,17 \%)$ were RAJl with significant EBNA1 KD shown (Table S3, Fig. 4A, B). Their identities were further verified by comprehensive STR analyses using 16 markers. 
One representative clone (BJAB:RAJI E1TN-selected-1 [BJRJ $\left.{ }^{\mathrm{ST}-1}\right]$ ) was presumed to be BJAB from the STR. As presumed, this clone was found to be BJAB from the comprehensive STR analyses (Fig. 4C). In addition, EBV negativity in BJRJ ${ }^{\mathrm{ST}-1}$ was shown through EBER in situ staining (Fig. 4D). EBNA1 KD was collinear to EBNA2 and LMP1 KD in representative clones (Fig. 3E). These spike experiments indicate that transient EBNA1 KO can selectively attenuate EBV-infected cells and counter select more EBV-negative cells in vitro via selectively targeting EBV + RAJl cells.

\section{E1TN induced progressive loss of EBV episome from EBV-infected BL cells}

We next attempted to determine the efficiency of E1TN pair to eliminate EBV episomes from the transformed BL cells whose growth depends on the presence of EBV. In support of this idea (20), we infected BJAB cells with green EBV BAC virus, in which the GFP represents the persistence of EBV episome persistence. We then transfected $\mathrm{RFP}^{+} / \mathrm{GFP}^{-@ E B N A 1}$ reporter into BJAB-EBV ${ }^{\mathrm{GFP}}$ cells along with or without the E1TN pair. The $\mathrm{RFP}^{+} / \mathrm{GFP}^{+}$cells were FACS-sorted and plated, and the $\mathrm{EBV}^{\mathrm{GFP}+}$ cell fraction was determined by flow cytometry. Single transient transfection of E1TN pair induced progressive loss of EBV episome from the EBV-infected BJAB BL cells (Fig. $4 \mathrm{~F}$ ). The progressive loss of EBV was presented with GFP (Note that transient single transfection induced EBV loss by $39 \%$ to $32 \%$ over 2 weeks upon transient KD of EBNA and the cells were not subjected to selection, as the E1TN pair carries no antibiotics resistance markers).

\section{DISCUSSION}

We attempted to eradicate EBV episome from EBV-infected GC, BL, and LCL cells by targeting EBNA1, an essential protein for latent viral replication and persistence. Our results showed that targeted transient disruption of EBNA1 by E1TN could induce selective death of naturally EBV-infected cells, but not EBV-negative cells, thereby resulting in no derivation of EBV-eliminated daughter cells from EBV-infected parental cells. These results strongly support the hypothesis that EBNA1-mediated EBV latent episome persistence is necessary for persistent growth of EBV-infected cells. The requirement of EBNA1 for EBV persistence was further substantiated in the subsequent spike experiments; the pre-mixture of EBV-infected RAJI cells with EBV-negative BJAB cells and subsequent targeted transient disruption of EBNA1 counter-selected mostly EBV-negative BJAB cells.

Knocking out multiple alleles of EBNA1 that are likely present in polyploidy cells would be highly difficult. Therefore, we have attempted multiple rounds of $\mathrm{KO}$ by repeated transfection of the E1TN pair. However, this was inadequate to acclaim any negative derivatives. From multiple rounds of E1TN pair transfection into several different EBV-infected cell lines in this study, more than 14 RAJl colonies managed to grow but none of them showed complete lack of EBV episomes or knockout of EBNA1, suggesting that cell death is followed by EBNA1 KO, because of the defects in EBV genome replication or persistence. Similar results were observed in SNU-265 and SNU-719 cells, supporting that whichever cells containing nuclease-induced mutations most likely died out.

To the best of our knowledge, this is the first report that TALEN-mediated transient targeting of EBNA1 can efficiently attenuate the growth of EBV-infected cells, implicating possible therapeutic application of E1TN when the E1TN pair was effectively delivered by concentrated nasal spray containing E1TN, in particularly to the nasopharyngeal epithelial mucosa in nasopharyngeal carcinoma patients. Because a TALEN system requires two TALEN vectors, use of lentivirus or adenovirus is not feasible to deliver two TALEN vectors in a single viral vector which has a small maximum cloning capacity. In summary, transient, targeted-disruption of EBNA1 induced death and growth attenuation of EBV-infected cells.

\section{MATERIALS AND METHODS}

\section{EBNA1 TALEN design}

To disrupt EBNA1, two EBNA1_TALEN nucleases (E1TN_L1 and E1TN_R1) were constructed: E1TN_L1 and E1TN_R1 recognize EBNA 1 nucleotide (nt) 41 to $5 \overline{9}$ from the start codon and nt 72 to 90 , respectively. Six different DNA binding domain (DBD) modules each of which recognizes 3 bases from the EBNA1 sequence nt 41 to 59 were selected, arrayed, and fused to the catalytic domain of type II restriction enzyme Fokl to induce specific cleavage of EBNA1 site (E1TN_L1). Similarly, 6 additional DBD modules that recognize 3 bases each from the nt 72 to 90 were fused to Fokl as well (E1TN R1) $(17,18)$. Additionally, a $\mathrm{RFP}^{+} / \mathrm{GFP}^{-@ E B N A 1}$ reporter plasmid that harbors two reporter genes(constitutively expressing the monomeric red fluorescence protein (RFP) open reading frame (ORF) and conditionally expressing the inducible enhanced green fluorescence protein (EGFP) ORF) with the target site (EBNA1 nt 40 to 90) fused to the GFP ORF out-of-frame was constructed. Constitutive RFP indicates transfection efficiency while EGFP expression implies that a potential target site was disrupted. EGFP only becomes expressed when the E1TN pair mediates in-frame correction from the out-of-frame site at upstream of GFP ORF. The most efficiently working target sites by EBNA1 TALEN were as follows; left TALEN (E1TN L) 19 bases target sequence is $5^{\prime}$-TAG GAC AGA AGG AAG ACT C-3' and right TALEN (E1TN R) 19 base target sequence is $5^{\prime}$-TCC ACT GCC GCC GGA GCC T-3'. In human, no such 19 base sequence with $100 \%$ identical to EBNA1 are found. The RFP ${ }^{+} / \mathrm{GFP}^{-@ E B N A 1}$ reporter plasmid contains an EBNA1 oligo sequence, that is 5'-CTA GGA CAG AAG GAA GAC TCA ACA GGA CCA GAA GGC TCC GGC GGC AGT GGA (A)-3'. 
Cell culture, transfection, sorting and selection of targeted cells, verification of gene disruption and rate of EBV genome loss upon E1TN introduction

EBNA1 gene disruption was verified by T7E1 assay as described $(17,18,20)$ (detail in SI).

\section{Spike experiments}

EBV-negative $B J A B$ cells were mixed with EBV-positive RAJI cells with at a ratio of 1:10, 1:100 and 1:1000 and transfected with a pair of TALEN plasmids ( $2 \mu \mathrm{g}$ each) and $\mathrm{RFP}^{+} / \mathrm{GFP}^{-@ E B N A 1}$ reporter plasmid $(4 \mu \mathrm{g})$. Two days later, double positive (RFP + GFP + cells) cells were sorted and plated at 10 or $10^{2}$ cells/well, and the number of surviving colonies was scored. EBNA1 KO was tested by T7E1 and western blotting, whilst the identity of overgrown colonies was verified by short tandem repeats (STR) profiling. For mini-STR, FGA STR marker was amplified using primer set of forward 5'-GGCTGCAGGGCATAA CATTA-3' and reverse 5'-ATTCTATGACTTTGCGCTTCAGGA$3^{\prime}$ and separated on $10 \%$ polyacrylamide/1XTAE gel. Complete DNA fingerprinting for 16 STR markers was undertaken at the Korea Cell Line Bank, a World Intellectual Property Organization (WIPO)-accredited international cell line repository institute (Seoul, Korea).

\section{ACKNOWLEDGEMENTS}

This study was supported by a grant from the National Research Foundation of Korea, NRF-2015 R1A2A2A01006459 granted to MSK and NRF-2014R1A1A2056910 to EKL. This work was supported by the National Research Foundation of Korea (NRF) grant funded by the Korean government (MSIP) (No. 2015048055).

\section{REFERENCES}

1. Parkin DM (2006) The global health burden of infectionassociated cancers in the year 2002. Int J Cancer 118, 3030-3044

2. Granato M, Feederle R, Farina A et al (2008) Deletion of Epstein-Barr virus BFLF2 leads to impaired viral DNA packaging and primary egress as well as to the production of defective viral particles. J Virol 82, 4042-4051

3. Adams A and Lindahl T (1975) Epstein-Barr virus genomes with properties of circular DNA molecules in carrier cells. Proc Natl Acad Sci U S A 72, 1477-1481

4. Raynaud FI, Whittaker SR, Fischer PM et al (2005) In vitro and in vivo pharmacokinetic-pharmacodynamic relationships for the trisubstituted aminopurine cyclin-dependent kinase inhibitors olomoucine, bohemine and CYC202. Clin Cancer Res 11, 4875-4887

5. Henderson A, Ripley S, Heller M and Kieff E (1983) Chromosome site for Epstein-Barr virus DNA in a Burkitt tumor cell line and in lymphocytes growth-transformed in vitro. Proc Natl Acad Sci U S A 80, 1987-1991

6. Matsuo T, Heller M, Petti L, O'Shiro E and Kieff E (1984)
Persistence of the entire Epstein-Barr virus genome integrated into human lymphocyte DNA. Science 226, 1322-1325

7. Sugden B, Marsh K and Yates J (1985) A vector that replicates as a plasmid and can be efficiently selected in B-lymphoblasts transformed by Epstein-Barr virus. Mol Cell Biol 5, 410-413

8. Yates JL, Warren N and Sugden B (1985) Stable replication of plasmids derived from Epstein-Barr virus in various mammalian cells. Nature 313, 812-815

9. Kang MS, Lee EK, Soni V et al (2011) Roscovitine inhibits EBNA1 serine 393 phosphorylation, nuclear localization, transcription, and episome maintenance. J Virol 85, 2859-2868

10. Kang MS, Hung SC and Kieff E (2001) Epstein-Barr virus nuclear antigen 1 activates transcription from episomal but not integrated DNA and does not alter lymphocyte growth. Proc Natl Acad Sci U S A 98, 15233-15238

11. Nasimuzzaman $M$, Kuroda $M$, Dohno $S$ et al (2005) Eradication of epstein-barr virus episome and associated inhibition of infected tumor cell growth by adenovirus vector-mediated transduction of dominant-negative EBNA1. Mol Ther 11, 578-590

12. Yin $Q$ and Flemington EK (2006) siRNAs against the Epstein Barr virus latency replication factor, EBNA1, inhibit its function and growth of EBV-dependent tumor cells. Virology 346, 385-393

13. Miller JC, Tan S, Qiao G et al (2011) A TALE nuclease architecture for efficient genome editing. Nat Biotechnol 29, 143-148

14. Kim SY, Song KA, Kieff E and Kang MS (2012) Small molecule and peptide-mediated inhibition of Epstein-Barr virus nuclear antigen 1 dimerization. Biochem Biophys Res Commun 424, 251-256

15. Lee EK, Kim SY, Noh KW et al (2014) Small molecule inhibition of Epstein-Barr virus nuclear antigen-1 DNA binding activity interferes with replication and persistence of the viral genome. Antiviral Res 104C, 73-83

16. Cohen JI, Mocarski ES, Raab-Traub N, Corey L and Nabel GJ (2013) The need and challenges for development of an Epstein-Barr virus vaccine. Vaccine 31 Suppl 2, B194-196

17. Kim Y, Kweon J, Kim A et al (2013) A library of TAL effector nucleases spanning the human genome. Nat Biotechnol 31, 251-258

18. Sung YH, Baek IJ, Kim DH et al (2013) Knockout mice created by TALEN-mediated gene targeting. Nat Biotechnol 31, 23-24

19. Sung YH, Baek IJ, Seong JK, Kim JS and Lee HW (2012) Mouse genetics: catalogue and scissors. BMB Rep 45, 686-692

20. Molesworth SJ, Lake CM, Borza CM, Turk SM and HuttFletcher LM (2000) Epstein-Barr virus gH is essential for penetration of $B$ cells but also plays a role in attachment of virus to epithelial cells. J Virol 74, 6324-6332

21. Park J, Bae EK, Lee C et al (2014) Establishment and characterization of bortezomib-resistant U266 cell line: constitutive activation of NF-kappaB-mediated cell signals and/or alterations of ubiquitylation-related genes reduce bortezomib-induced apoptosis. BMB Rep 47, 274-279 